\title{
Strong anisotropy of the electron-phonon interaction in NbP probed by magnetoacoustic quantum oscillations
}

\author{
Clemens Schindler $®,{ }^{1,2,}{ }^{*}$ Denis Gorbunov, ${ }^{3}$ Sergei Zherlitsyn, ${ }^{3}$ Stanislaw Galeski, ${ }^{1}$ Marcus Schmidt, ${ }^{1}$ \\ Jochen Wosnitza, ${ }^{2,3}$ and Johannes Gooth ${ }^{1,2, \dagger}$ \\ ${ }^{1}$ Max Planck Institute for Chemical Physics of Solids, 01187 Dresden, Germany \\ ${ }^{2}$ Institut für Festkörper- und Materialphysik, Technische Universität Dresden, 01062 Dresden, Germany \\ ${ }^{3}$ Hochfeld-Magnetlabor Dresden (HLD-EMFL) and Würzburg-Dresden Cluster of Excellence ct.qmat, Helmholtz-Zentrum \\ Dresden-Rossendorf, 01328 Dresden, Germany
}

(Received 21 August 2020; accepted 13 October 2020; published 30 October 2020)

\begin{abstract}
In this study, we report on the observation of de Haas-van Alphen-type quantum oscillations (QOs) in the ultrasound velocity of $\mathrm{NbP}$ as well as "giant QOs" in the ultrasound attenuation in pulsed magnetic fields. The difference in the QO amplitude for different acoustic modes reveals a strong anisotropy of the effective deformation potential, which we estimate to be as high as $9 \mathrm{eV}$ for certain parts of the Fermi surface. Furthermore, the natural filtering of QO frequencies and the tracing of the individual Landau levels to the quantum limit allows for a more detailed investigation of the Fermi surface of NbP, as was previously achieved by means of analyzing QOs observed in magnetization or electrical resistivity.
\end{abstract}

DOI: 10.1103/PhysRevB.102.165156

\section{INTRODUCTION}

Probing the propagation of ultrasound in the quantum regime of electrons yields detailed information on the nature of electron-phonon interactions. The ultrasound velocity in such a regime exhibits quantum oscillations (QOs), which can be understood both from a thermodynamic argument [1,2] and from a self-consistent treatment of ultrasound propagation as a stream of acoustic phonons interacting with an electron gas that is quantized into Landau levels (LLs) [3-6]. Both approaches yield the same result, namely, the amplitude of the QOs being dependent on the (effective) deformation potential $\Xi_{i}^{k}=d E_{k} / d \varepsilon_{i}$, which is a measure of the change in energy $E_{k}$ of an electronic band $k$ at a given strain $\varepsilon_{i}$. The connection to the microscopic picture can be understood intuitively by recalling that the probability for an electron in the $k$ th band to be scattered by a phonon-mode corresponding to $\varepsilon_{i}$ is proportional to $\left(\Xi_{i}^{k}\right)^{2}$ [3-9]. Employing measurements of magnetoacoustic QOs, the deformation potential and its anisotropy have been experimentally determined for many metals and semimetals (see, for example, Refs. [3,9-14]).

Recently, the semimetallic transition-metal monopnictide $\mathrm{NbP}$ has been of great interest, mainly due to its symmetryprotected crossings of conduction and valence bands which potentially host Weyl fermions [15-17]. It exhibits a very

\footnotetext{
*clemens.schindler@cpfs.mpg.de

$\dagger$ johannes.gooth@cpfs.mpg.de
}

Published by the American Physical Society under the terms of the Creative Commons Attribution 4.0 International license. Further distribution of this work must maintain attribution to the author(s) and the published article's title, journal citation, and DOI. Open access publication funded by the Max Planck Society. small and highly anisotropic Fermi surface, consisting of intercalated spin-split pairs of electron and hole pockets due to spin-orbit coupling [18]. The small Fermi surface gives rise to pronounced QOs of relatively low frequencies, which have so far been observed in magnetization [18-20], electrical resistivity [21-24], Hall resistivity [21,23], thermal conductivity [19], thermopower [19], and heat capacity [19]. The superposition of QOs originating from different extremal Fermisurface orbits yields a rich Fourier spectrum, especially when $\boldsymbol{H}$ is aligned along the $c$ axis of the tetragonal lattice and the extremal orbits are the smallest. The peaks in the Fourier spectra could be assigned to orbits via comparison of experimental data to $a b$ initio density functional theory (DFT) calculations [18], however, ambiguities due to the limited resolution and the broadness of the Fourier peaks remained. In a recent study by some of the authors [23], the evolution of the Fermi surface upon direct application of uniaxial stress along the $a$ axis has been probed by means of Shubnikov-de Haas $(\mathrm{SdH})$ oscillations in the electrical resistivity. These experiments revealed a strong strain dependence of the $\mathrm{SdH}$ oscillations, which, besides the additional information regarding the orbit assignments, also render NbP a promising platform for studying magnetoacoustic QOs. Furthermore, the strong anisotropy of the Fermi surface is suggestive of a highly anisotropic electron-phonon interaction as well, which can be most conveniently investigated via ultrasonic measurements.

In this paper, we report on the measurements of QOs in the ultrasound velocity and attenuation in a NbP single crystal in pulsed magnetic fields $\boldsymbol{H} \| c$ (or [001]). We have investigated the acoustic modes $(u\|q\|[100]),(u\|q\|[001]),(u \|$ [001], $q \|$ [100]), $(u\|[010], q\|[100])$, and $(u\|[1 \overline{10}], q\|$ [110]) corresponding to the elastic moduli $C_{11}, C_{33}, C_{44}, C_{66}$, and $\left(C_{11}-C_{12}\right) / 2$ (using Voigt notation). Here, $u$ is the displacement vector, and $q$ is the direction of propagation of the acoustic wave. Significant differences of the individual QO 
amplitudes between the modes were revealed. A large signalto-noise ratio, the usage of pulsed magnetic fields beyond the quantum limit, the high quality of our sample resulting in peak-shaped QOs (the presence of higher harmonics of the Fourier series), and the natural filtering of certain QO frequencies due to the anisotropic electron-phonon interaction allowed for a detailed analysis of the QO frequencies and amplitude ratios. Thereby, the anisotropy of $\Xi_{i}^{k}$ and partially also the cyclotron masses, cyclotron mobilities, and phase factors for several extremal Fermi-surface orbits were determined. The QO frequency spectrum could be analyzed via direct assignments of the LL peaks rather than Fourier analysis as in previous studies, which allowed for the assignment of formerly elusive orbits. In addition, the extremal nature (maximum or minimum) of the individual orbits could be deduced from the asymmetric shape of the LL peaks.

\section{METHODS}

$\mathrm{NbP}$ has a tetragonal crystal lattice (space group $I 4{ }_{1} m d$, No. 109) with the lattice parameters $a=b=3.3324(2) \AA$ and $c=11.13705(7) \AA$ [25]. A single-crystalline sample of $\mathrm{NbP}$ was grown using chemical vapor transport reactions; the sample was also used in our previous work [23] for the determination of the elastic moduli. For acoustic modes propagating along one of the main axes, the sample was cut accordingly to a cuboid shape of dimensions $1.92 \times 1.80 \times$ $0.88 \mathrm{~mm}^{3}$. For the $\left(C_{11}-C_{12}\right) / 2$ mode, two cuts parallel to the (110) plane were subsequently added. The crystal planes were carefully polished, and two lithium niobate $\left(\mathrm{LiNbO}_{3}\right)$ transducers ( $Z$ cut for longitudinal waves and $X$ cut for transverse waves) were glued to opposite parallel surfaces for excitation and detection of acoustic waves. The relative ultrasound-velocity changes $\Delta v / v$ and attenuation changes $\Delta \alpha$ were measured using an ultrasound pulse-echo phasesensitive detection technique $[9,26]$ in pulsed magnetic fields up to $38 \mathrm{~T}$ (test pulses up to $56 \mathrm{~T}$ ) at temperatures ranging from 1.35 to $30 \mathrm{~K}$. Excitation frequencies were varied from 27 to $100 \mathrm{MHz}$ with pulse durations ranging from 50 to $200 \mathrm{~ns}$. Strain-induction coupling, i.e., the Alpher-Rubin effect [2], may be safely neglected at the used frequencies as the large magnetoresistance in $\mathrm{NbP}$ even at moderate magnetic fields $\left(\mu_{0} H>1 \mathrm{~T}\right)$ prevents a strong skin effect.

\section{RESULTS}

The change in sound velocity $\Delta v / v$ and the change in sound attenuation $\Delta \alpha$ vs magnetic field at $T=1.35 \mathrm{~K}$ are shown for different acoustic modes in Fig. 1. Here, $\Delta v / v$ refers to the change compared to the sound velocity at zero magnetic field $v=\sqrt{C_{\text {eff }} / \rho}$, where $C_{\text {eff }}$ is the effective elastic constant governing the respective mode [27] and $\rho$ is the mass density $\left(\rho=6.52 \mathrm{~g} \mathrm{~cm}^{-3}\right.$ for NbP [25]). $\Delta v / v$ shows pronounced QOs with high harmonic content, whereas dominant frequencies and the size of the oscillation amplitudes strongly vary between the modes. Strikingly, the QO amplitude in the $C_{66}$ mode is smaller by a factor of $\approx 20$ compared to the other modes, where for the last few LL changes in $v$ by more than one part in a thousand are observed. $\Delta \alpha$ exhibits QOs with a characteristic spikelike shape, also varying in terms of

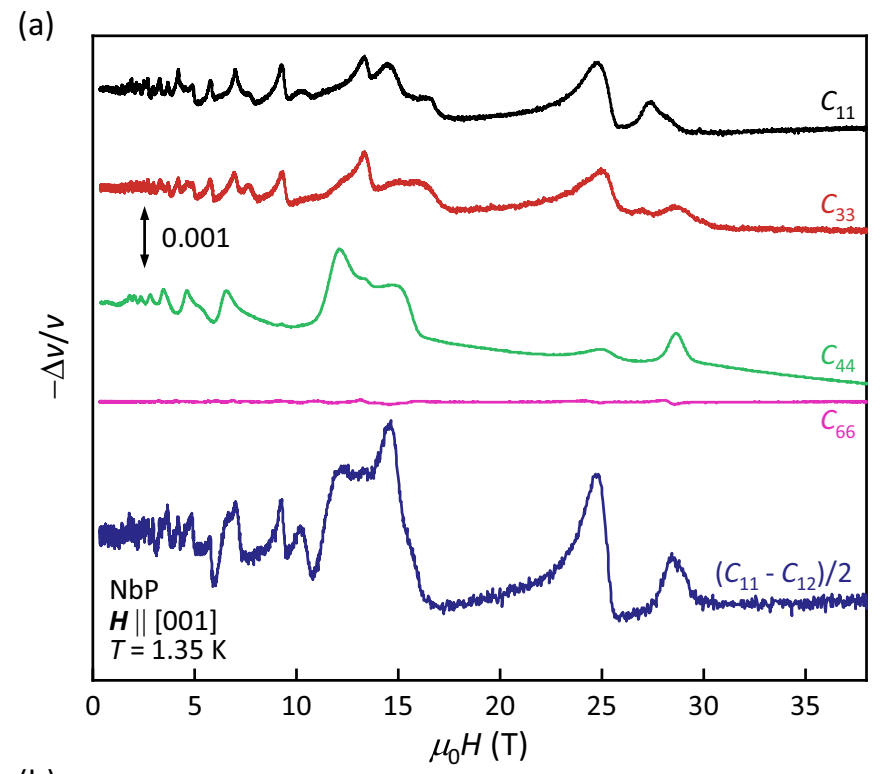

(b)

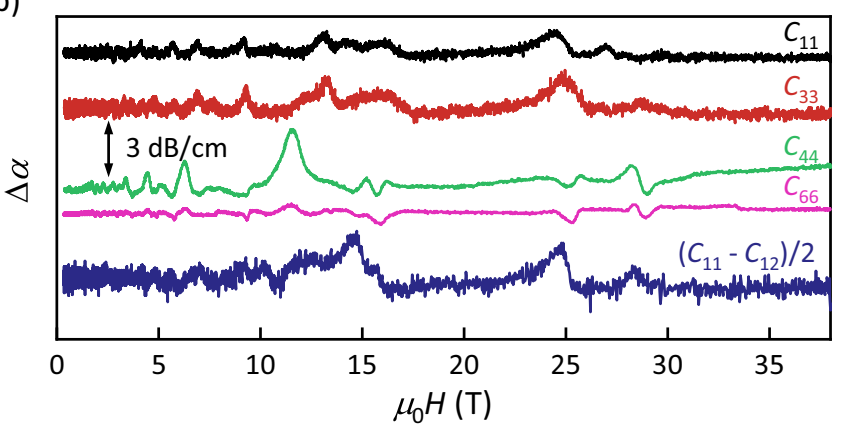

FIG. 1. Magnetoacoustic quantum oscillations in NbP for pulsed magnetic fields $\boldsymbol{H} \| c$ at $T=1.35 \mathrm{~K}$ for different acoustic modes. (a) Change in the relative ultrasound velocity $-\Delta v / v$ versus magnetic field. (b) Change in ultrasound attenuation $\Delta \alpha$ versus magnetic field. The curves are shifted with respect to each other for better visibility.

amplitude and dominant frequencies depending on the mode. We recall that the physical mechanism responsible for the QOs in ultrasound attenuation, which are commonly termed "giant QOs" [1,8], is not related to the Landau tubes passing through the extremal parts of the Fermi surface as in the de Haas-van Alphen (dHvA)-type oscillations. Instead, spikes in $\Delta \alpha$ occur when the Landau tubes pass through the Fermisurface section, where the component of the Fermi velocity parallel to $q$ is equal to the phase velocity of sound $[1,3,8,28]$. This resonance condition is the reason for the spikelike shape, as it is fulfilled only for particular values of the wave vector, in contrast to the contribution of many wave vectors in the dHvA-type oscillations. Notably, the resonant Fermi-surface orbits can differ substantially from the extremal orbits, especially when $q \perp \boldsymbol{H}$. Hence, the positions of the observed spikes in $\Delta \alpha$ do not necessarily coincide with the LL peaks in $\Delta v / v$.

Above $30 \mathrm{~T}$, all electrons and holes are confined to their lowest LL, and $v(H)$ and $\alpha(H)$ exhibit a steady slope in the investigated field (measured up to $56 \mathrm{~T}$ for $C_{44}$ ) and temperature range, showing no signatures for correlation-driven charge instabilities. Such correlation-driven phase transitions, 
e.g., a charge density wave, would manifest in a slope change in $\Delta v / v$ and a peak in $\Delta \alpha$ [29] and have been predicted to occur in the extreme quantum limit of Weyl semimetals [30,31]. Notably, there have been observations of indicative features in the extreme quantum limit in the electrical resistivity and in the sound velocity and attenuation in the related compound TaAs [32,33]. However, in the case of pristine $\mathrm{NbP}$ the interaction strength presumably is too feeble to allow for experimental access to these energy scales within our achievable field and temperature range.

\section{A. Quantum oscillations in the velocity of sound}

\section{Frequency analysis and orbit assignment}

To analyze the QOs in the ultrasound velocity, $-\Delta v / v$ is plotted against $1 / H$ (Fig. 2). The ultrasound velocity, just like any thermodynamic property of a material, exhibits singularities upon increasing magnetic field whenever a cyclotron orbit corresponding to a LL is exactly equal to an extremal orbit of the Fermi-surface sheet perpendicular to the applied $\boldsymbol{H}$. According to the Onsager relation [1], these singularities are periodic in $1 / H$ with frequency $F=(\hbar / 2 \pi e) A_{\text {ext }}$, where $A_{\text {ext }}$ is the area enclosed by the corresponding extremal orbit, $\hbar$ is the reduced Planck constant, and $e$ is the electron charge. Plotting the LL number vs $1 / H, F$ can then be extracted using a linear fit [see Fig. 2(g)].

For a maximum orbit, $-\Delta v / v$ will increase with $(1 / H)^{-1 / 2}$ approaching a LL singularity from a lower field and then decrease steeply once the area of the corresponding cyclotron orbit exceeds that of the maximum orbit [28]. Accordingly, for a minimum orbit these slopes are reversed, and the steep rise appears on the low-field side of the LL peak. If smearing due to finite temperature and electron scattering is sufficiently suppressed, the QOs retain a high harmonic content and approach a sawtoothlike shape. The asymmetry of the individual LL peaks then allows for identifying whether the corresponding peak is arising from a maximum or minimum orbit of the Fermi surface.

Clearly, the dominant frequency of $30.89 \mathrm{~T}$ in $C_{11}$ and $C_{33}$ [also very well distinguishable in the $\left(C_{11}-C_{12}\right) / 2$ mode] stems from a maximum orbit [most apparent for the last three LLs; see Fig. 2(b)]. It is also the most pronounced frequency in the $\mathrm{SdH}$ oscillations in magnetoresistance [Fig. 2(b) top], whose shape resembles that of the $C_{11}$ mode. As assigned in Ref. [18] based on DFT calculations and further indicated by comparing experimental and calculated strain dependences [23], this frequency likely stems from the $\alpha_{1}$ orbit rather than the $\gamma_{1}$ orbit [hereafter, we use the same labeling for the extremal orbits of $\mathrm{NbP}$ as in these Refs. [18,23]; see Fig. 2(a)]. The $\alpha_{1}$ oscillation is much less pronounced in $C_{44}$ [see Fig. 2(c)], allowing for clear identification of the 14.74-T oscillation as a minimum orbit, assigned to $\beta_{1}$. After having identified the LL peaks for $\alpha_{1}$ and $\beta_{1}$, the remaining peaks in the high-field range might be assigned to the $\gamma_{1}$ orbit and possibly also the $\delta_{1}$ orbit [see Fig. 2(e)]. The assignment to $\delta_{1}$ is rather speculative; the second peak at approximately $0.06 \mathrm{~T}^{-1}$ might also stem from the last LL of $\delta_{2}$. At low fields, a 0.9-T oscillation with minimum-orbit characteristics is visible in $C_{44}$, assigned to $\beta_{2}$ [Fig. 2(d)]. Furthermore, by applying a low-pass Fourier filter to $C_{11}$ an oscillation of
6.81 $\mathrm{T}$ is singled out, which was also identified in the Fourier spectra from previous QO studies [18,22,23] and assigned to the $\alpha_{2}$ orbit [Fig. 2(f)]. The extracted frequencies are summarized in Table I. We note that we did not observe additional QO patterns predicted to occur in Weyl semimetals when the Fermi level is near the Weyl points [5].

\section{Lifshitz-Kosevich fit}

The actual shape of the QOs in $\Delta v / v$ can be described by a Fourier series taking finite-temperature smearing of the Fermi-Dirac distribution and LL broadening due to electron scattering into account. After Lifshitz and Kosevich [1], the oscillatory part of $\Delta v / v$ for a single QO frequency without spin degeneracy holds:

$$
\begin{aligned}
\frac{\tilde{v}_{i j}}{v_{i j}}= & -\frac{1}{2}\left(\frac{\partial F}{\partial \varepsilon_{i}}\right)\left(\frac{\partial F}{\partial \varepsilon_{j}}\right) \frac{e^{2} V}{m_{\mathrm{c}} C_{i j}}\left(\frac{2 e H}{\hbar \pi^{3} A_{\mathrm{ext}}^{\prime \prime}}\right)^{\frac{1}{2}} \\
& \times \sum_{p=1}^{\infty} p^{-\frac{1}{2}} R_{T} R_{\mathrm{D}} \cos \left[2 \pi p\left(\frac{F}{H}-\varphi\right) \pm \frac{\pi}{4}\right]
\end{aligned}
$$

where $m_{\mathrm{c}}$ denotes the effective cyclotron mass, $V$ is the realspace volume, $A_{\text {ext }}^{\prime \prime}$ is the curvature of the Fermi surface at the extremal orbit, and $\varphi$ is the phase factor. The $\pm \pi / 4$ phase shift accounts for whether the orbit is maximum $(-)$ or minimum $(+)$. Damping of the QOs due to thermal smearing of the Fermi distribution is accounted for by the factor [1]

$$
R_{\mathrm{T}}=\frac{\lambda(T)}{\sinh [\lambda(T)]}, \quad \lambda(T)=p \frac{2 \pi^{2} m_{\mathrm{c}} k_{\mathrm{B}} T}{e \hbar H} .
$$

Damping due to electron scattering is taken into account by the Dingle damping factor [1]

$$
R_{\mathrm{D}}=\exp \left[-\lambda\left(T_{\mathrm{D}}\right)\right]=\exp \left[-p \frac{\pi}{\mu_{\mathrm{c}} H}\right],
$$

where $T_{\mathrm{D}}$ is the Dingle temperature and $\mu_{\mathrm{c}}$ is the mobility of an electron exerting cyclotron motion in an applied magnetic field (not to be confused with the zero-field transport mobility, which, depending on the current direction, can significantly differ from $\mu_{\mathrm{c}}$ in the case of a large band anisotropy [35]). The $\beta_{1}, \beta_{2}$, and $\alpha_{1}$ oscillations were clearly distinguishable in $C_{44}$ and $C_{33}$, respectively, and could be approximated using the first 20 harmonics of Eq. (1). From fits to the QOs for different temperatures (Fig. 3), the damping factors $R_{\mathrm{D}}$ and $R_{T}$ could be extracted, allowing for the determination of $m_{\mathrm{c}}, \varphi, \mu_{\mathrm{c}}$, and $T_{\mathrm{D}}$ (summarized in Table I). The fitting procedure was performed globally for all temperatures with the shared parameters $F$ (fixed), $m_{\mathrm{c}}, \varphi$, and $\mu_{\mathrm{c}}$ and an independent amplitude prefactor. We note that the direct fitting of the naturally filtered QOs yields a greater reliability for the $m_{\mathrm{c}}$ values compared to the analysis of Fourier spectra, as there the field-dependent amplitude damping usually leads to a systematic underestimation of $m_{\mathrm{c}}[23,36]$. Our fits yielded an effective cyclotron mass of $0.06(1) m_{0}$ for $\alpha_{1}$ and $0.12(2) m_{0}$ for $\beta_{1}$, which is larger than the values extracted from Fourier analysis of dHvA oscillations [18] [0.047(9) $m_{0}$ and 0.057(7) $\left.m_{0}\right]$. The extracted $m_{\mathrm{c}}$ are also in better agreement with the calculated values from Ref. [18] $\left(0.10 m_{0}\right.$ and $\left.0.12 m_{0}\right)$ compared to previous methods, although this does not necessarily imply improved accuracy. 
(a)
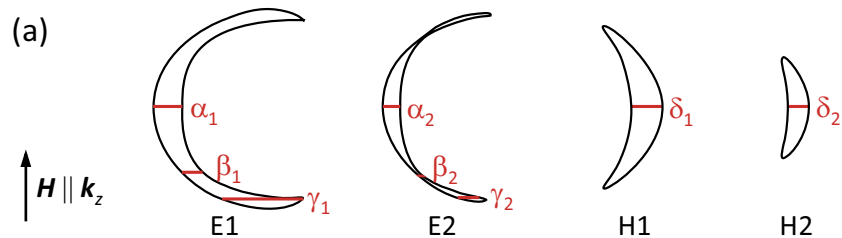

$\mathrm{H} 1$

$\mathrm{H} 2$

(b)

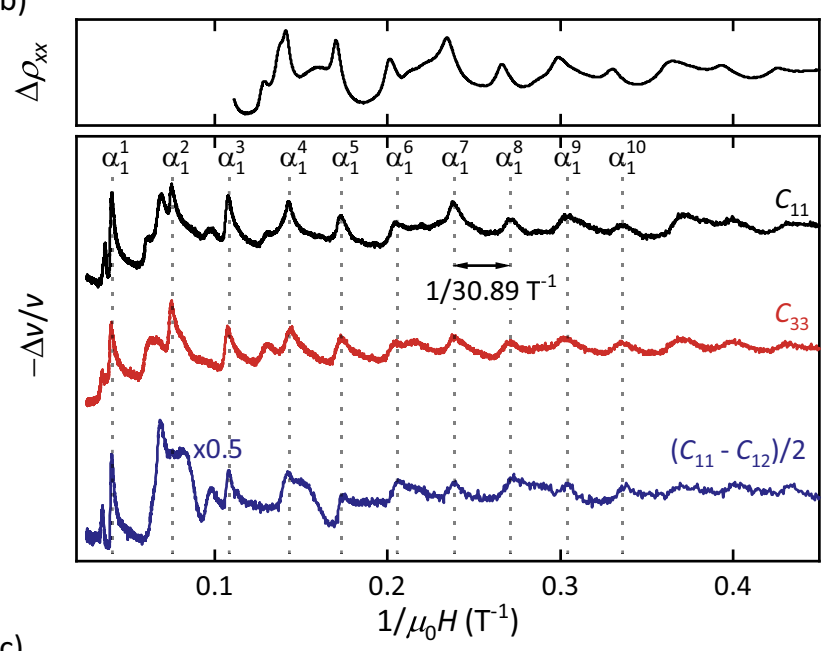

(c)

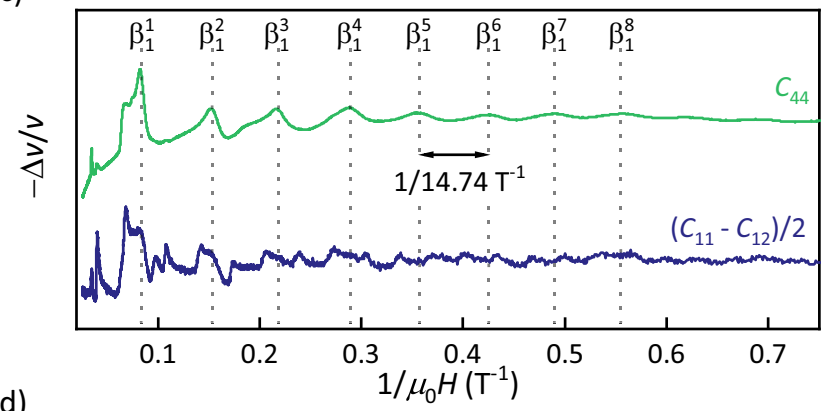

(d)

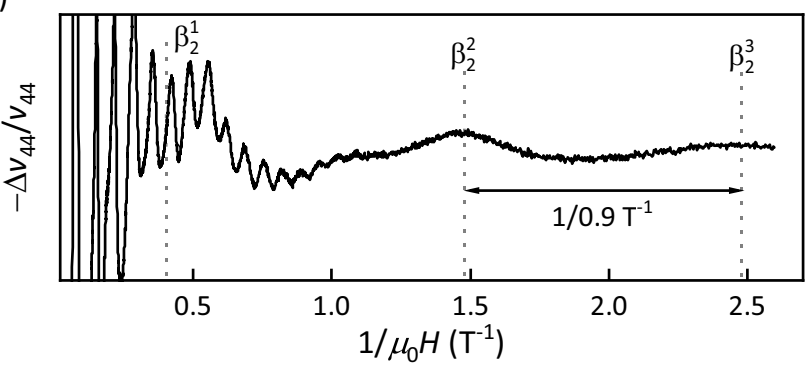

(e)

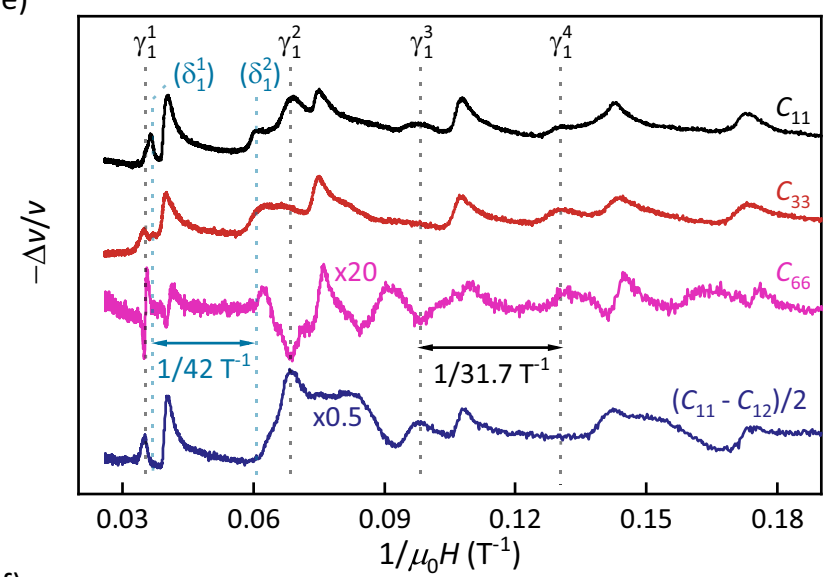

(f)

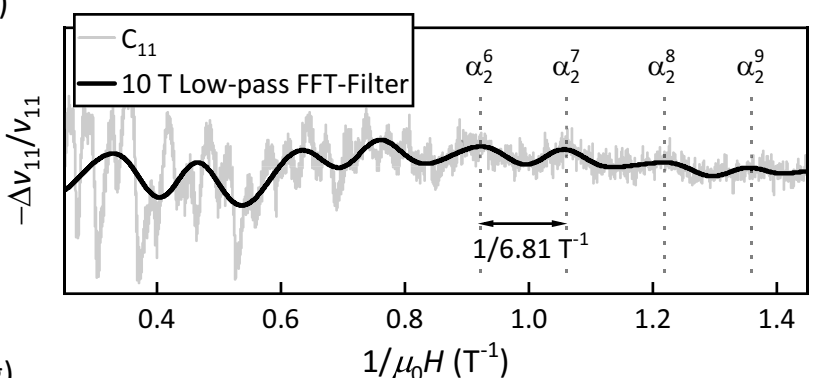

(g)

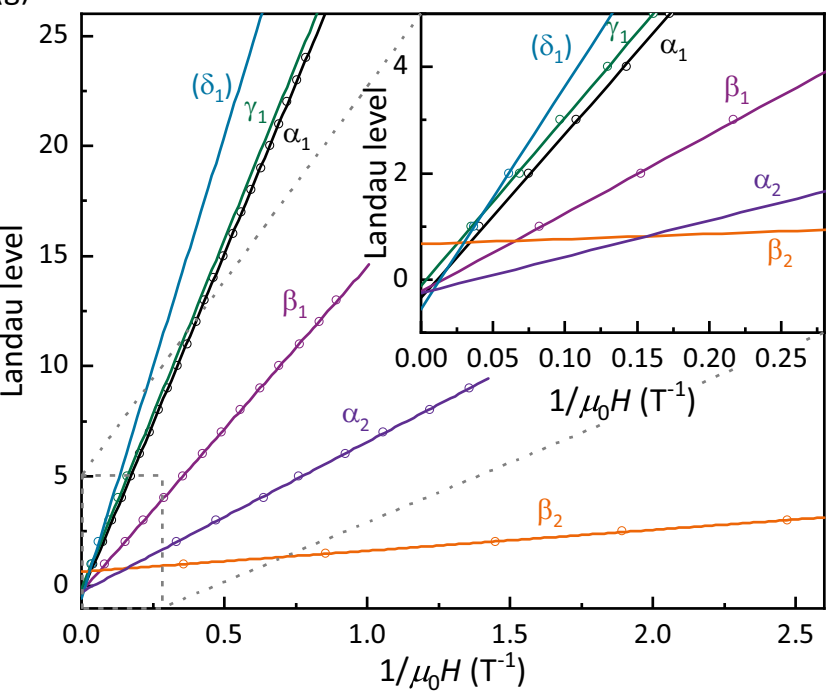

FIG. 2. Frequency analysis of the quantum oscillations in ultrasound velocity for different modes at $T=1.35 \mathrm{~K}$. (a) Projections of the electron pockets, $\mathrm{E} 1$ and $\mathrm{E} 2$, and the hole pockets, $\mathrm{H} 1$ and $\mathrm{H} 2$, of $\mathrm{NbP}$ parallel to the $k_{x}-k_{z}$ plane (or, similarly, to the $k_{y}-k_{z}$ plane due to the fourfold rotational symmetry). Extremal orbits for $\boldsymbol{H} \| c$ are shown in red. For an illustration of the full Fermi surface in the first Brillouin zone see, for instance, Refs. [23,34]. (b) Top: Shubnikov-de Haas oscillations subtracted from the magnetoelectrical resistivity $\rho_{x x}$ at $T=2 \mathrm{~K}$ for comparison. Bottom: Landau level peaks assigned to the maximum orbit $\alpha_{1}$. (c) Landau level peaks assigned to the minimum orbit $\beta_{1}$. (d) Low-frequency oscillation visible in the $C_{44}$ mode assigned to the minimum orbit $\beta_{2}$. (e) Assignment of the remaining peaks in the high-field range to the second maximum orbit of E1, $\gamma_{1}$, and possibly the maximum orbit $\delta_{1}$. (f) Oscillation assigned to the maximum orbit $\alpha_{2}$ visible in the $C_{11}$ mode, emphasized by applying a low-pass Fourier filter. (g) Assigned Landau levels plotted versus inverse magnetic field. Solid lines represent linear fits. The inset enlarges the high-field range.

\section{Discussion of the phase factor}

The phase factors extracted from fitting Eq. (1) to the $\Delta v / v$ data are around 0.5 for the extremal orbits $\alpha_{2}$ and $\beta_{2}$ on electron pocket E2 and vary from 0.27 to 0.20 for the orbits $\alpha_{1}, \beta_{1}$, and $\gamma_{1}$ on E1. According to recent theoretical works by Alexandradinata et al. [37,38], the phase factor generally consists of three contributions:

$$
\varphi=\varphi_{\mathrm{M}}-\varphi_{\mathrm{B}}-\varphi_{\mathrm{d}},
$$

where $\varphi_{\mathrm{M}}$ is the Maslov correction $\left(\varphi_{\mathrm{M}}=1 / 2\right.$ for orbits that are compressible to a circle, which is the case for all orbits in $\mathrm{NbP}) ; \varphi_{\mathrm{B}}$ is the geometric phase, i.e., Berry phase [39], that 
TABLE I. Experimental results extracted from the analysis of quantum oscillations in the ultrasound velocity for $\boldsymbol{H} \| c$. The calculated orbits are denoted as in Ref. [18], and experimentally extracted frequencies $F$ are assigned as in Refs. [18,23], considering also the asymmetry of the Landau level peaks due to the extremal nature of the orbit (maximum or minimum). The cyclotron mass $m_{\mathrm{c}}$, cyclotron mobility $\mu_{\mathrm{c}}$, Dingle temperature $T_{\mathrm{D}}$, phase factor $\varphi$, and effective deformation potential $\Xi_{i}$ with respect to $\Xi_{1}$ are given where possible (only absolute values). $\Xi_{\mathrm{s}}$ denotes the deformation potential corresponding to the $\left(C_{11}-C_{12}\right) / 2$ mode. Extr. refers to Extremal nature.

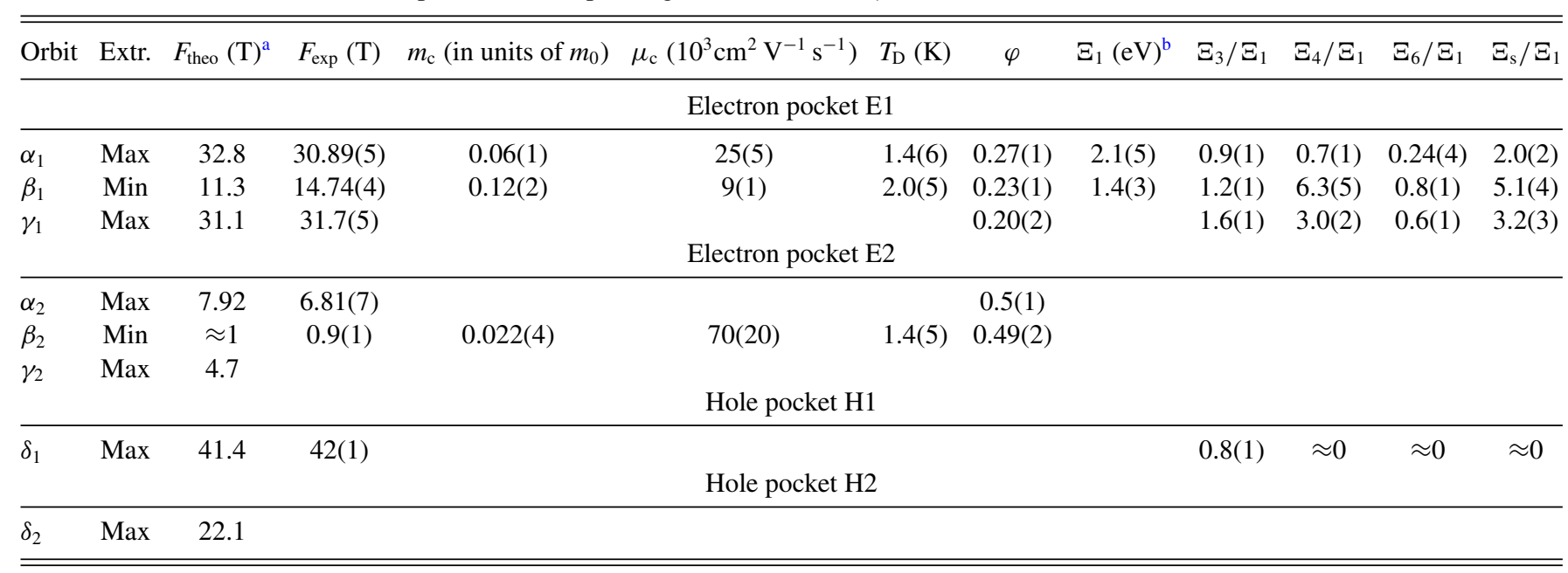

a The calculated frequencies were obtained from density functional theory in our previous study [23].

${ }^{\mathrm{b}} \Xi_{1}$ has been estimated with Eq. (5) using the averaged $\partial F / \partial \varepsilon_{1}$ values from Ref. [23].

an electron acquires upon encircling the orbit in reciprocal space; and $\varphi_{\mathrm{d}}$ is the dynamic phase factor which accounts for the generalized Zeeman interaction of the intrinsic and orbital magnetic moment. The main interest in analyzing the phase contributions lies in the extraction of $\varphi_{\mathrm{B}}$, as it potentially allows us to identify topologically nontrivial bands, such as Weyl and Dirac bands [39]. Indeed, under certain symmetry constraints (for details, see Refs. [37,38]) $\varphi_{\mathrm{d}}$ vanishes or can only take quantized values $\pm 1 / 2$, which then allows us to draw conclusions about $\varphi_{\mathrm{B}}$. As all orbits in $\mathrm{NbP}$ for $\boldsymbol{H} \| c$ can be mapped onto themselves in $k$ space upon applying a mirror operation (mirror planes $k_{x}=0$ or $k_{y}=0$, see Ref. [34]), they belong to the classification (II-A, $u=1, s=0$ ) of Table I in Ref. [37], and $\varphi_{\mathrm{d}}$ can be either 0 or $1 / 2$ depending (a)

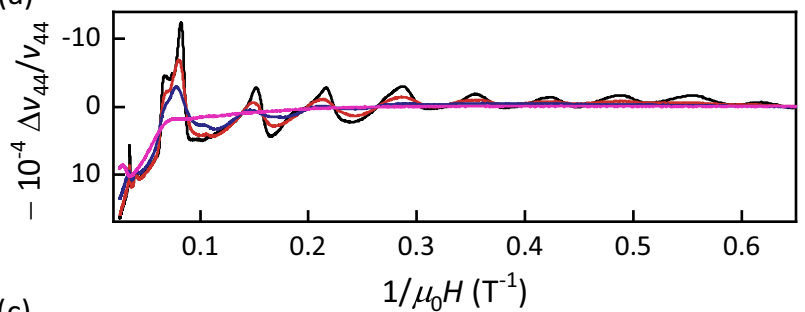

(c)

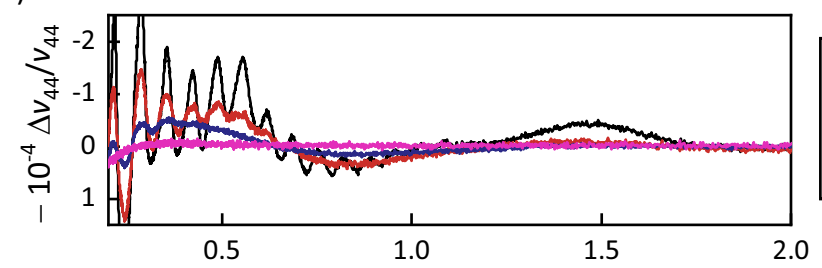

(e)

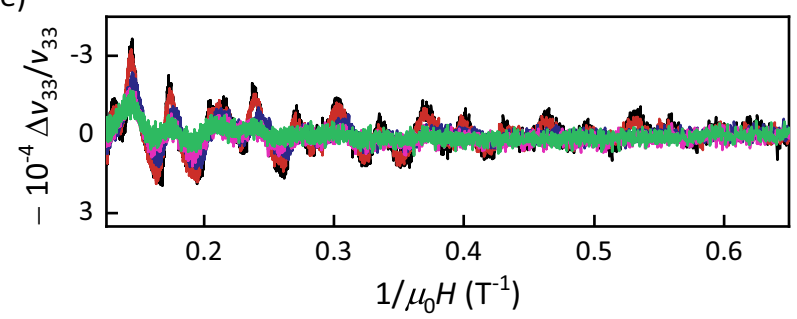

(b)

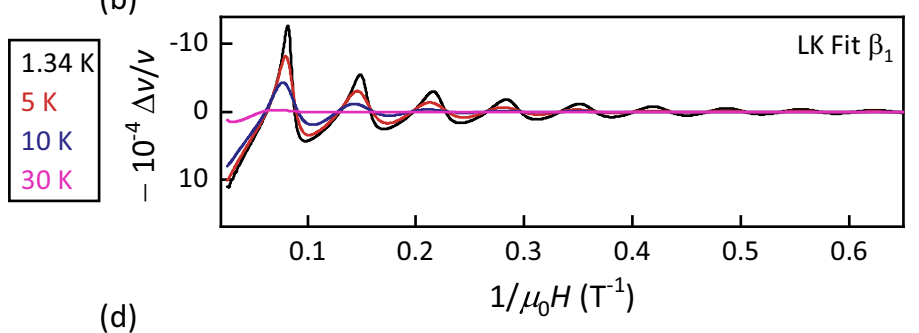

(d)
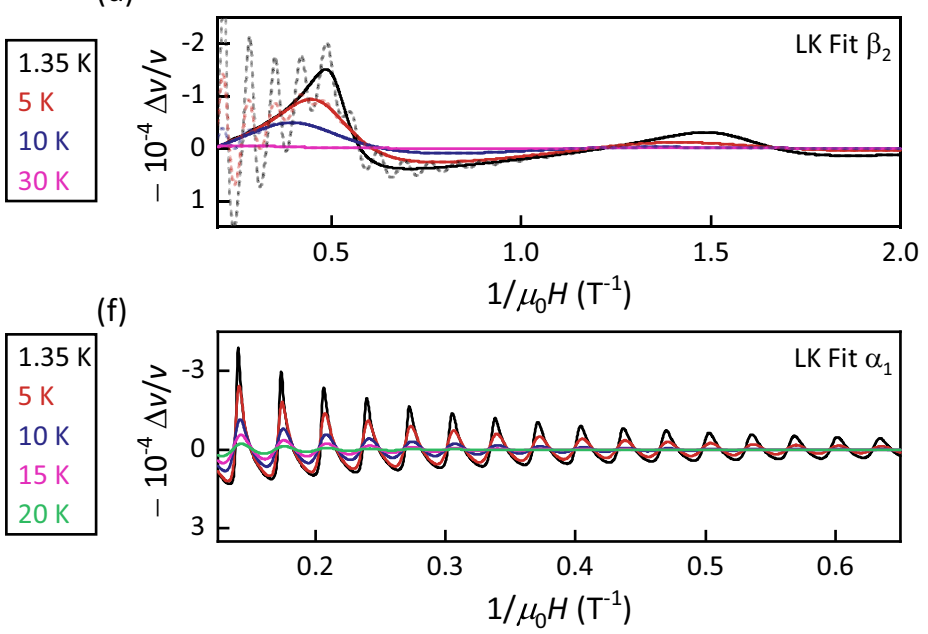

FIG. 3. Temperature evolution of the quantum oscillations in the ultrasound velocity for the (a) and (c) $C_{44}$ and (e) $C_{33}$ modes and LifshitzKosevich fit for frequencies (b) $\beta_{1}$, (d) $\beta_{2}$, and (f) $\alpha_{1}$ dominant in $C_{44}$ and $C_{33}$, respectively. 
(a)

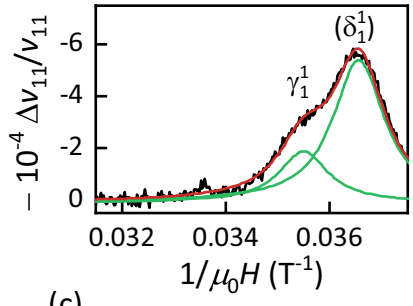

(c)

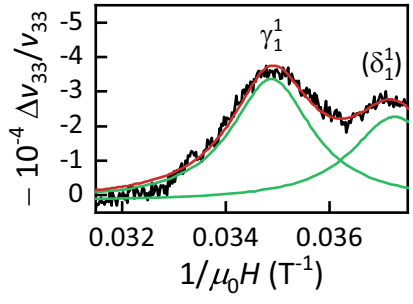

(b)

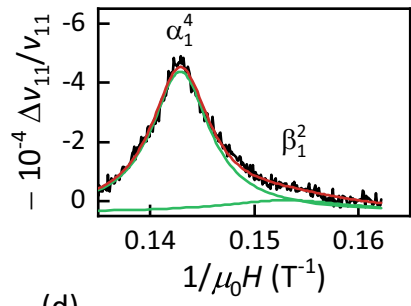

(d)

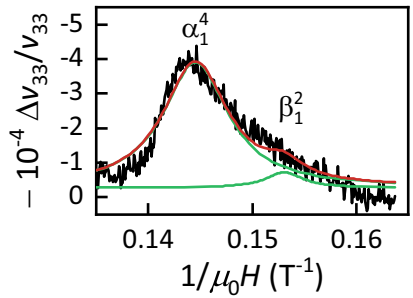

FIG. 4. Extraction of the oscillation amplitude for superimposed peaks in the high-field range by fitting two Lorentzian functions (green) at fixed inverse-field values. Extraction of the height for $\gamma_{1}^{1}$ and $\delta_{1}^{1}$ from the ultrasonic quantum oscillations in the (a) $C_{11}$ and (c) $C_{33}$ modes. Extraction of the height for $\beta_{1}^{2}$ for the (b) $C_{11}$ and (d) $C_{33}$ modes.

on details of the band structure. Hence, at first glance it may seem that a deviation of $\varphi$ from 0 or $1 / 2$ can be regarded as a signature of a non-zero $\varphi_{\mathrm{B}}$; However, it was shown by Klotz et al. [18] that the Fermi-surface pockets in NbP intersecting with the Weyl bands, E1 and H1, always encompass a pair of Weyl points and should thus exhibit a trivial phase shift of $\varphi_{\mathrm{B}}=1$ or 0 . Hence, the extracted phase factors of $\alpha_{1}$, $\beta_{1}$ and $\gamma_{1}$ are at odds with the possible values predicted by theory. It is rather speculative why that is the case, the reason might be slight misalignment of the magnetic field, wrong orbit assignment or, more generally, inaccuracy of the DFT calculations, although the latter two are highly improbable given the otherwise good agreement. The extracted $\varphi$ of E2 do not contradict theory, but are also not particularly informative regarding the topological nature of the bands.

\section{Extraction of the deformation potentials}

Comparing the amplitudes of the same orbit for different modes, the ratio of the $C_{i i}^{-1}\left(d F / d \varepsilon_{i}\right)^{2}$ values can be extracted. With the known elastic constants from our previous study [23], the ratio of the effective deformation potentials can then be calculated via [2]

$$
\Xi_{i}=\frac{d E}{d \varepsilon_{i}}=\frac{d E}{d A_{\mathrm{ext}}} \frac{d A_{\mathrm{ext}}}{d \varepsilon_{i}}=\frac{\hbar e}{m_{\mathrm{c}}} \frac{\partial F}{\partial \varepsilon_{i}} .
$$

The amplitude ratios for the individual orbits have been extracted by selecting easily distinguishable LL peaks (near the quantum limit) and dividing their top-to-bottom heights. In the case with no separate LL peak, for example, for the $\beta_{1}$ orbit in $C_{11}$ and $C_{33}$, the height was estimated by fitting two Lorentzian functions with fixed centers (Fig. 4), whereas the center positions were extracted from comparison with other modes (see Fig. 2). The resulting deformation potentials with respect to $\Xi_{1}$ are summarized in Table I. They are strongly anisotropic - measurable $\Xi$ values vary by up to a factor of $\approx 8$ depending on the direction of strain-which reflects the

(a)

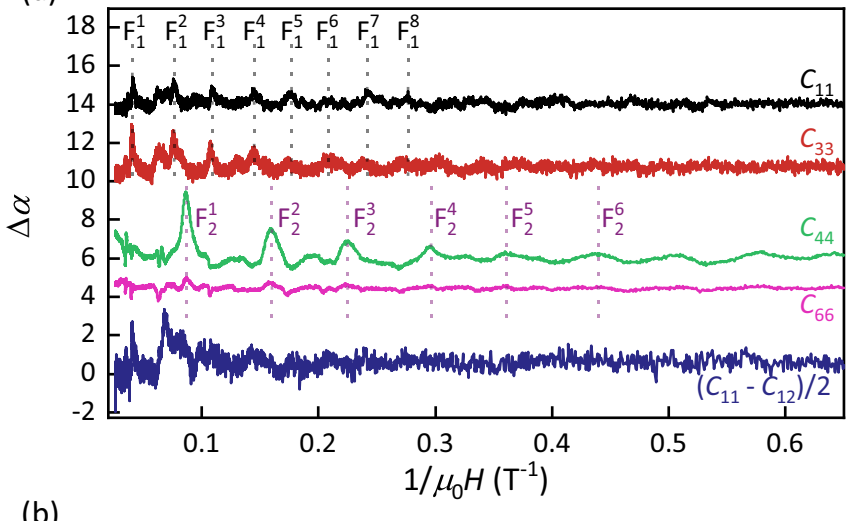

(b)

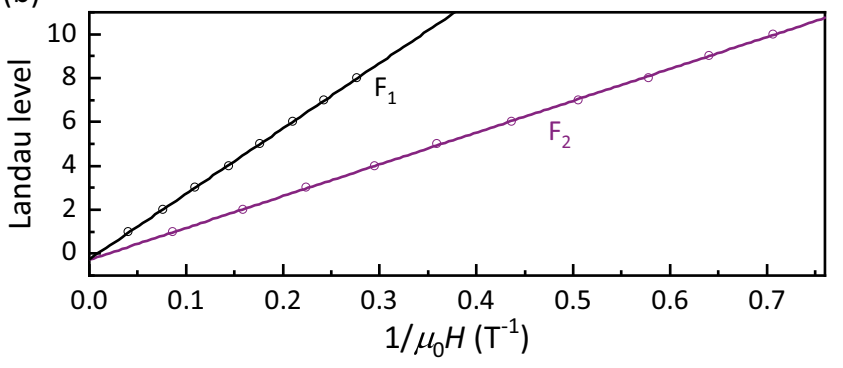

FIG. 5. Frequency analysis of the giant quantum oscillations in ultrasound attenuation for different modes at $T=1.35 \mathrm{~K}$. (a) Landau level peaks assigned to the resonant orbits $F_{1}$ and $F_{2}$. (b) Assigned Landau levels plotted vs inverse magnetic field. Solid lines represent linear fits.

anisotropy of the electronic bands in NbP (see DFT calculations in Refs. [34,40]). In contrast to the isotropic behavior in conventional metals, the electron-phonon scattering in $\mathrm{NbP}$ [and, transferably, other (Weyl) semimetals with anisotropic bands] is highly selective.

With the $\partial F / \partial \varepsilon_{1}$ values gathered from Ref. [23], $\Xi_{1}$ can be estimated via Eq. (5) to be $2.1 \mathrm{eV}(2.5 \mathrm{eV})$ for $\alpha_{1}$ and $1.4 \mathrm{eV}$ $(2.2 \mathrm{eV})$ for $\beta_{1}$, taking experimental (calculated) values. For $\beta_{1}$, this results in an effective deformation potential of $9 \mathrm{eV}$ $(14 \mathrm{eV})$ for shear strain along $c$. This potential is among the highest reported values $[10,41,42]$ and illustrates how electrons in the narrow part of the electron pocket are extremely susceptible to interaction with phonon modes corresponding to such shear strain. We note that upon applying strain along an axis perpendicular to the $c$ axis, the breaking of the rotational symmetry leads to a degeneracy lifting of the Fermi pockets and $\partial F / \partial \varepsilon_{1}$ actually splits into a positive branch and a negative branch [23]. As in Eq. (1) the sign of $\partial F / \partial \varepsilon_{1}$ is canceled due to the square; we took the average of the absolute values in order to estimate $\Xi_{1}$.

\section{B. Giant quantum oscillations in ultrasound attenuation}

The giant QOs in $\Delta \alpha$ are less straightforward to analyze, as the position of the resonant orbits in reciprocal space is rather complicated to determine for each corresponding phonon mode. If plotted against $1 / H$ [Fig. 5(a)], two periodic series of spikes are very clearly distinguishable, labeled $F_{1}$ and $F_{2}$. The Onsager relation is valid for the giant QOs as well; linear fits to the spike positions vs LL number yield $F_{1}=29.8 \mathrm{~T}$ and $F_{2}=14.5 \mathrm{~T}$ [Fig. 5(b)]. The areas enclosed 
by the resonant orbits are thus close to those of $\alpha_{1}$ and $\beta_{1}$. A puzzling feature is the observation of the same frequencies in two modes with perpendicular $q$, e.g., $F_{1}$ in both $C_{11}$ and $C_{33}$. This observation might be explained by the peculiar shape of the Fermi surface in NbP, where fourfold-degenerate sicklelike pockets are located near the edges of the first Brillouin zone. In this particular case, the resonant condition might be fulfilled for the same orbit for elastic waves propagating along both $a$ and $c$.

In contrast to the QO in $\Delta v / v$, the exact shape of the spikes in $\Delta \alpha$ is rather difficult to fit. Each $\delta$ function corresponding to a spike must be convoluted with various distribution functions accounting for the effects of finite temperature and electron scattering [1]. In our case, this did not seem viable as multiple frequencies superimpose on each other, and similar information on the electronic properties was already extracted from the QOs in $\Delta v / v$, where the signal-to-noise ratio was also more favorable. Nevertheless, the slight asymmetry of the spikes can be attributed to an indirect effect of electron scattering, where the smearing of the LL relaxes the resonance condition [1]. The spikes of $F_{1}$ and $F_{2}$ are broader towards the low-field side [see Fig. 1(b)], which is indicative of a convex curvature of the Fermi surface at the resonant orbit $\left(A^{\prime \prime}<0\right)$.

\section{SUMMARY}

In summary, we studied the QOs in ultrasound velocity and attenuation in NbP in pulsed magnetic fields. Fields with $\boldsymbol{H} \| c$ beyond the quantum limit were applied. We compared the QOs for several acoustic modes, revealing significant dif- ferences regarding which orbits are dominant. By extracting the amplitudes of the QOs in the ultrasound velocity, the anisotropy of the deformation potentials was determined for several extremal orbits. A large deformation potential of approximately $9 \mathrm{eV}$ for the minimum orbit $\beta_{1}$ under shear strain along the $c$ axis was revealed, suggesting that electrons in this part of the Fermi surface are very susceptible to interactions with the phonon modes corresponding to $C_{44}$. Furthermore, the high harmonic content of the QOs and the large field range allowed for a more reliable determination of the frequencies, effective cyclotron masses, and mobilities, as was previously achieved by means of Fourier analysis. As a side note, we did not find any signatures for correlated electron states in the quantum limit of (pristine) NbP.

\section{ACKNOWLEDGMENTS}

C.S. would like to thank A. Alexandradinata for engaging in helpful discussions. C.S. acknowledges financial support from the International Max Planck Research School for Chemistry and Physics of Quantum Materials (IMPRS-CPQM). The work was supported by Deutsche Forschungsgemeinschaft (DFG) through SFB 1143 and the Würzburg-Dresden Cluster of Excellence on Complexity and Topology in Quantum Matter (ct.qmat; EXC 2147, Project No. 390858490) and by Hochfeld-Magnetlabor Dresden (HLD) at HZDR, a member of the European Magnetic Field Laboratory (EMFL).
[1] D. Shoenberg, Magnetic Oscillations in Metals (Cambridge University Press, Cambridge, 1984).

[2] L. R. Testardi and J. H. Condon, 2-Landau quantum oscillations of the velocity of sound and the strain dependence of the Fermi surface, Phys. Acoust. 8, 59 (1971).

[3] Y. Shapira, 1-Acoustic wave propagation in high magnetic fields, Phys. Acoust. 5, 1 (1968).

[4] M. Kataoka and T. Goto, Theory of the acoustic de Haas-van Alphen effect, J. Phys. Soc. Jpn. 62, 4352 (1993).

[5] S.-B. Zhang and J. Zhou, Quantum oscillations in acoustic phonons in Weyl semimetals, Phys. Rev. B 101, 085202 (2020).

[6] J. Mertsching, Theorie elektromagnetischer Wellen in Metallen und ihrer Wechselwirkung mit Ultraschallwellen (I), Phys. Status Solidi B 14, 3 (1966).

[7] J. Bardeen and W. Shockley, Deformation potentials and mobilities in non-polar crystals, Phys. Rev. 80, 72 (1950).

[8] V. L. Gurevich, V. L. Skobov, and Y. A. Firsov, Giant quantum oscillations in the acoustical absorption by a metal in a magnetic field, J. Expt. Theor. Phys. (U.S.S.R.) 40, 786 (1961) [Sov. Phys. JETP 13, 552 (1961)]

[9] B. Lüthi, Physical Acoustics in the Solid State, Springer Series in Solid-State Sciences (Springer, Berlin, 2006).

[10] K. Walther, Anisotropy of magnetoacoustic attenuation and deformation potential in bismuth, Phys. Rev. 174, 782 (1968).
[11] T. E. Thompson, P. R. Aron, B. S. Chandrasekhar, and D. N. Langenberg, Magnetostriction and magnetoelastic quantum oscillations in $p-$ PbTe, Phys. Rev. B 4, 518 (1971).

[12] H. Matsui, T. Goto, M. Kataoka, T. Suzuki, H. Harima, S. Kunii, R. Takayama, and O. Sakai, Acoustic de Haas-van Alphen effect of $\mathrm{LaB}_{6}$, J. Phys. Soc. Jpn. 64, 3315 (1995).

[13] J. M. V. Martins, F. P. Missell, and J. R. Pereira, Strain dependence of the Fermi surface in cadmium and rhenium from ultrasonic velocity oscillations, Phys. Rev. B 17, 4633 (1978).

[14] J. Nössler, R. Seerig, S. Yasin, M. Uhlarz, S. Zherlitsyn, G. Behr, S.-L. Drechsler, G. Fuchs, H. Rosner, and J. Wosnitza, Field-induced gapless electron pocket in the superconducting vortex phase of $\mathrm{YNi}_{2} \mathrm{~B}_{2} \mathrm{C}$ as probed by magnetoacoustic quantum oscillations, Phys. Rev. B 95, 014523 (2017).

[15] H. Weng, C. Fang, Z. Fang, B. A. Bernevig, and X. Dai, Weyl Semimetal Phase in Noncentrosymmetric TransitionMetal Monophosphides, Phys. Rev. X 5, 011029 (2015).

[16] S.-Y. Xu, I. Belopolski, N. Alidoust, M. Neupane, G. Bian, C. Zhang, R. Sankar, G. Chang, Z. Yuan, C.-C. Lee, S.-M. Huang, H. Zheng, J. Ma, D. S. Sanchez, B. Wang, A. Bansil, F. Chou, P. P. Shibayev, H. Lin, S. Jia, and M. Z. Hasan, Discovery of a Weyl fermion semimetal and topological Fermi arcs, Science 349, 613 (2015). 
[17] S.-M. Huang, S.-Y. Xu, I. Belopolski, C.-C. Lee, G. Chang, B. K. Wang, N. Alidoust, G. Bian, M. Neupane, C. Zhang, S. Jia, A. Bansil, H. Lin, and M. Z. Hasan, A Weyl fermion semimetal with surface Fermi arcs in the transition metal monopnictide TaAs class, Nat. Commun. 6, 7373 (2015).

[18] J. Klotz, S.-C. Wu, C. Shekhar, Y. Sun, M. Schmidt, M. Nicklas, M. Baenitz, M. Uhlarz, J. Wosnitza, C. Felser, and B. Yan, Quantum oscillations and the Fermi surface topology of the Weyl semimetal NbP, Phys. Rev. B 93, 121105(R) (2016).

[19] U. Stockert, R. D. dos Reis, M. O. Ajeesh, S. J. Watzmann, M. Schmidt, C. Shekhar, J. P. Heremans, C. Felser, M. Baenitz, and M. Nicklas, Thermopower and thermal conductivity in the Weyl semimetal NbP, J. Phys.: Condens. Matter 29, 325701 (2017).

[20] P. Sergelius, J. Gooth, S. Bäßler, R. Zierold, C. Wiegand, A. Niemann, H. Reith, C. Shekhar, C. Felser, B. Yan, and K. Nielsch, Berry phase and band structure analysis of the Weyl semimetal NbP, Sci. Rep. 6, 33859 (2015).

[21] C. Shekhar, A. K. Nayak, Y. Sun, M. Schmidt, M. Nicklas, I. Leermakers, U. Zeitler, Y. Skourski, J. Wosnitza, Z. Liu, Y. Chen, W. Schnelle, H. Borrmann, Y. Grin, C. Felser, and B. Yan, Extremely large magnetoresistance and ultrahigh mobility in the topological Weyl semimetal candidate NbP, Nat. Phys. 11, 645 (2015).

[22] R. D. dos Reis, S. C. Wu, Y. Sun, M. O. Ajeesh, C. Shekhar, M. Schmidt, C. Felser, B. Yan, and M. Nicklas, Pressure tuning the Fermi surface topology of the Weyl semimetal NbP, Phys. Rev. B 93, 205102 (2016).

[23] C. Schindler, J. Noky, M. Schmidt, C. Felser, J. Wosnitza, and J. Gooth, Effect of uniaxial stress on the electronic band structure of NbP, Phys. Rev. B 102, 035132 (2020).

[24] A. C. Niemann, J. Gooth, S.-C. Wu, S. Bäßler, P. Sergelius, R. Hühne, B. Rellinghaus, C. Shekhar, V. Süß, M. Schmidt, C. Felser, B. Yan, and K. Nielsch, Chiral magnetoresistance in the Weyl semimetal NbP, Sci. Rep. 7, 43394 (2017).

[25] J. Xu, M. Greenblatt, T. Emge, P. Höhn, T. Hughbanks, and Y. Tian, Crystal structure, electrical transport, and magnetic properties of niobium monophosphide, Inorg. Chem. 35, 845 (1996).

[26] B. Wolf, B. Lüthi, S. Schmidt, H. Schwenk, M. Sieling, S. Zherlitsyn, and I. Kouroudis, New experimental techniques for pulsed magnetic fields - ESR and ultrasonics, Phys. B (Amsterdam, Neth.) 294-295, 612 (2001).

[27] K. Brugger, Pure modes for elastic waves in crystals, J. Appl. Phys. 36, 759 (1965).

[28] J. Mertsching, Theory of electromagnetic waves in metals and their interaction with ultrasonic waves (III), Phys. Status Solidi B 37, 465 (1970).

[29] D. LeBoeuf, C. W. Rischau, G. Seyfarth, R. Küchler, M. Berben, S. Wiedmann, W. Tabis, M. Frachet, K. Behnia, and B.
Fauqué, Thermodynamic signatures of the field-induced states of graphite, Nat. Commun. 8, 1337 (2017).

[30] M. Trescher, E. J. Bergholtz, M. Udagawa, and J. Knolle, Charge density wave instabilities of type-II Weyl semimetals in a strong magnetic field, Phys. Rev. B 96, 201101(R) (2017).

[31] M. Laubach, C. Platt, R. Thomale, T. Neupert, and S. Rachel, Density wave instabilities and surface state evolution in interacting Weyl semimetals, Phys. Rev. B 94, 241102(R) (2016).

[32] B. J. Ramshaw, K. A. Modic, A. Shekhter, Y. Zhang, E.-A. Kim, P. J. W. Moll, M. D. Bachmann, M. K. Chan, J. B. Betts, F. Balakirev, A. Migliori, N. J. Ghimire, E. D. Bauer, F. Ronning, and R. D. McDonald, Quantum limit transport and destruction of the Weyl nodes in TaAs, Nat. Commun. 9, 2217 (2018).

[33] C.-L. Zhang, B. Tong, Z. Yuan, Z. Lin, J. Wang, J. Zhang, C.-Y. Xi, Z. Wang, S. Jia, and C. Zhang, Signature of chiral fermion instability in the Weyl semimetal TaAs above the quantum limit, Phys. Rev. B 94, 205120 (2016).

[34] C.-C. Lee, S.-Y. Xu, S.-M. Huang, D. S. Sanchez, I. Belopolski, G. Chang, G. Bian, N. Alidoust, H. Zheng, M. Neupane, B. Wang, A. Bansil, M. Z. Hasan, and H. Lin, Fermi surface interconnectivity and topology in Weyl fermion semimetals TaAs, TaP, NbAs, and NbP, Phys. Rev. B 92, 235104 (2015).

[35] A. B. Pippard, Magnetoresistance in Metals, Cambridge Studies in Low Temperature Physics (Cambridge University Press, Cambridge, 1989).

[36] A. Audouard and J.-Y. Fortin, Does Fourier analysis yield reliable amplitudes of quantum oscillations? Eur. Phys. J. Appl. Phys. 83, 30201 (2018).

[37] A. Alexandradinata, C. Wang, W. Duan, and L. Glazman, Revealing the Topology of Fermi-Surface Wave Functions from Magnetic Quantum Oscillations, Phys. Rev. X 8, 011027 (2018).

[38] A. Alexandradinata and L. Glazman, Semiclassical theory of Landau levels and magnetic breakdown in topological metals, Phys. Rev. B 97, 144422 (2018).

[39] G. P. Mikitik and Y. V. Sharlai, Manifestation of Berry's Phase in Metal Physics, Phys. Rev. Lett. 82, 2147 (1999).

[40] Y. Sun, S.-C. Wu, and B. Yan, Topological surface states and Fermi arcs of the noncentrosymmetric Weyl semimetals TaAs, TaP, NbAs, and NbP, Phys. Rev. B 92, 115428 (2015).

[41] V. J. Tekippe, H. R. Chandrasekhar, P. Fisher, and A. K. Ramdas, Determination of the deformation-potential constant of the conduction band of silicon from the piezospectroscopy of donors, Phys. Rev. B 6, 2348 (1972).

[42] I. Vurgaftman, J. R. Meyer, and L. R. Ram-Mohan, Band parameters for III-V compound semiconductors and their alloys, J. Appl. Phys. 89, 5815 (2001). 